\title{
Emerging nanohybrid materials for chemical, biological, and engineering applications
}

\author{
Special Editors \\ Mita Dasog \\ Dalhousie University \\ Halifax, Nova Scotia \\ Robert W.J. Scott \\ University of Saskatchewan \\ Saskatoon, Saskatchewan \\ Jonathan G.C. Veinot \\ University of Alberta \\ Edmonton, Alberta
}

